\title{
A Study on Clinically Relevant Treatment Strategies for Nail Infections
}

\author{
Pratiksha Mishra \\ Shree Guru Gobind Singh Tricentary University, Gurugram, Haryana, India \\ Corresponding author email: pratiksha_fmhs@sgtuniversity.org
}

\section{ABSTRACT}

Fungal infection on nails is most underestimated by human beings due to lack of physical inconvenience apart from unconventional appearance of nail surface. Dermatophytes, yeasts and molds are major class of fungal pathogens responsible for nail infections, clinically called onychomycosis. These infections slowly progress into severe form deteriorating nail core by deposition of fungal spores which are unaffected by topical antifungal medicines. The disease takes a long time to heal by conventional procedure of topical treatments. The preset study explains onychomycosis from pathological perspective, reviewing the clinically relevant treatment strategies. Due to limitations in terms of antifungal drug penetration and patients' hesitation towards laser therapies, the study provides an unexplored research horizon and need for developing device based strategies for treatment of onychomycosis.

KEY WORDS: ANTI-FUNGAL, DERMATOPHYTES, LASER, NAIL INFECTIONS, ONYCHOMYCOSIS.

\section{INTRODUCTION}

Among several complex diseases prevailing in the world such as cancer, diabetes, skin infections, a mere yellowish appearance on a finger or toe nails becomes unnoticed or insignificant. These unusual appearances on finger nails are often observed in case of fungal infections. The fungal infection on the nails is known as tinea unguium or onychomycosis. The infection often remains undiagnosed due to lack of severe pain or uneasiness. The deficiency of health care services in developing countries like India, are responsible for growing prevalence of onychomycosis. The infection is usually characterized by appearance of unusual morphologies in the form of distorted, deformed, discolored, thickened nails with bent edges over nails of fingers or toes. The critical form of infection can cause separation of nail from the nail bed or migration of fungal species beyond nails, spreading areas between toes and skin of the foot leading to a disease called athlete's foot or tinea pedis. The disease is characterized by itching, stinging and burning sensation

Biosc Biotech Res Comm P-ISSN: 0974-6455 E-ISSN: 2321-4007
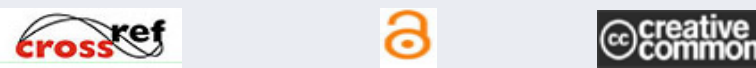

Identifiers and Pagination

Article Information

Year: 2021 Vol: 14 No (7) Special Issue

Pages: 283-287

Received: $08^{\text {th }}$ May 2021

This is an open access article under Creative

Commons License Attribn 4.0 Intl (CC-BY).

DOI: $h t t p: / / d x . d o i . o r g / 10.21786 / b b r c / 14.7 .62$ over rash developing on the feet's skin. Some form of nail infections can be left untreated; however severe disease form is treated with compounds targeting causative agents of onychomycosis.

Dermatophytes are most common etiological agent of onychomycosis but yeasts and non-dermatophyte molds have been reported to contribute to some of the onychomycosis cases. Specifically, T. rubrum are mostly reported as causative agents. Non dermatophytes such as Scytalidium dimidiatum produce disease patterns including other molds as causative agents such as Fusarium oxysporum, Scopulariopsis brevicaulis, Aspergillus spp, and Acremonium spp(A. K. Gupta (2001), A.K. Gupta (1996)). Onychomycosis caused by candida species usually lack distorted appearance of nails and known to affect the finger nails. Non-dermatophyte agents cause $6 \%$ of infections under the case of onychomycosis, commonly observed in old age patients with a history of some form of injury (S. Adekhandi et. al. (2015)). Risk factors associated with the disease include family history, age, moist and warm climate, occlusive shoes and traumas to nails associated with fungal invasion (B.E. Elewski et. al. (2015)). In order to treat onychomycosis, long treatments are usually prescribed which may extend from few months up to a year, where complete nail clearing or mycological disinfection is hard to achieve. 
Figure 1: Morphological characteristics and identification methods for nail fungus or onychomycosis. a) Hperkertosis of onychomycotic toe nail infected with fungal strain b) Microscopic image of Trichophyton rubrumone, one of the causative agent of onychomycosis. Trichophyton tonsurans (c) andAspergillus niger(d)observed onsample culture over Sabouraud dextrose agar(M. Ghannoum (2014), D.P. Westerberg (2013)

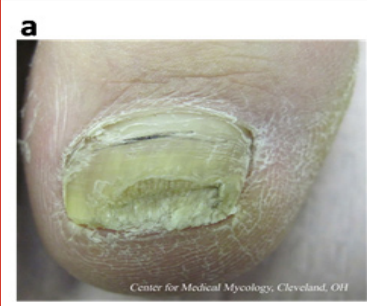

c

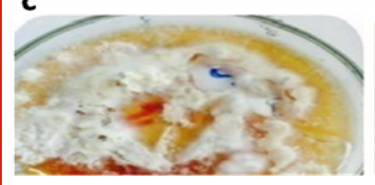

b

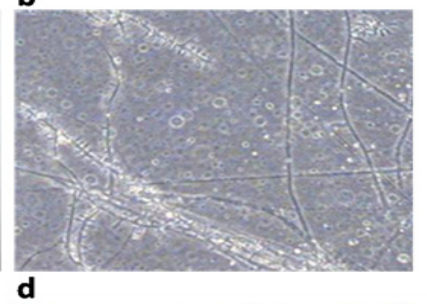

d

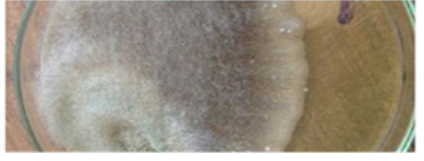

Pathological manifestations of onychomycosis: The morphological characteristics in onychomycosis resemble to several other nail infections, which can cause false positives while diagnosis of the disease. The accurate diagnosis is essential for prescribing treatments and involves examination of physical changes in the nail along with laboratory microbiological analysis. The disease suspicion begins by identification of discoloured, deformed or hyperkeratotic nail which is sterilized and then samples as nail clippings, debris are obtained for microscopic
Figure 2: The histological image of nail plate diagnosed with onychomycosis(D.P. Westerberg et. al. (2013)). a) Fusarium species detected by chlamydospores (arrows). b) Aspergillus species with characteristic hyphae braches (arrow) c) Candida species with budding spores (arrow) d) Undiagnosed fungal species of ubiquitous environmental mold Scedosporium apiospermum.

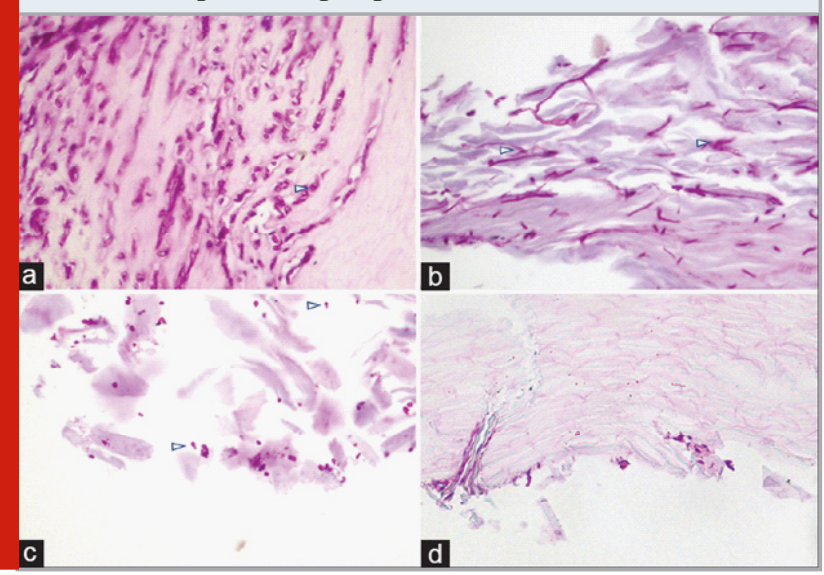

examination. The microscopic examination includes dissolving the keratin from nail clipping samples and using lactophenol cotton blue staining for detecting dermatophytes (E.E. din A. Nada et. al. (2020)). Subsequently, Sabouraud dextrose agar is used for microbial culturing to detect presence of cadida species. Candida differential agar can also be used for identification of candida species present in the nail samples. The microbiological in-vitro analysis is followed by histological studies using acid-Schiff staining. The staining method detects polysaccharides, glycogen, glycoprotein, glycolipids and mucins in the tissue.

\begin{tabular}{|c|c|c|}
\hline Nail lacquers & Action mechanism & Adverse effects \\
\hline Ciclopirox (Penlac 8\%) & $\begin{array}{l}\text { Chelation of cations and disruption } \\
\text { of fungal metabolic processes }\end{array}$ & Rashes, irritation, ingrown nail \\
\hline Amorolfine (5\%) & $\begin{array}{l}\text { Fungicidal effect - } \\
\text { inhibition of sterol synthesis }\end{array}$ & Itching, burning, erythma, scaling \\
\hline Econazole $(5 \%)$ & $\begin{array}{l}\text { Increased cellular permeability, } \\
\text { inhibition of cell membrane synthesis }\end{array}$ & Itching, stinging, redness \\
\hline
\end{tabular}

According to a study conducted by Lin et al., the staining method has been used to locate fungal components present in nails. Figure 1 shows histological examination of fungal species present in nail plates as characteristic causative agents for onychomycosis. The presence of Fusarium species have been marked by presence of chlamydospores. Aspergillus spieces has been identified by branched hyphae observed in Figure 2. Candida species has been identified by presence of budding spores. The study includes only the patients with positive diagnostic results and did not compare false negative results. The presence of the fungal elements can also result from contamination; hence proper control should have been used for comparison. The comparison should have been done with false positive samples having nail morphology similar to onychomychosis.

\section{Treatments for onychomycosis:}

2.1. Topical antifungal therapy: Nail lacquers or solutions are the most common forms of topical treatments for onychomycosis. Until 2014, ciclopirox has been used to treat infections caused by dermatophytes and yeast, also regarded as the only topical antifungal drug approved by the US FDA (United States Food and Drug Administration). However, clinical efficacy of the drug suffered due to 
difficulties in penetrating the nail plate (J.N. Queller et. al. (2015)). Moreover, most nail lacquers develop adverse reaction on figure tissue surrounding the nail (Table 1). Several in vitro studies have been performed to increase efficiency of ciclopirox penetration using oil based diffusion enhancers. Another study has reported that ciclopirox functional activity gets reduced in the presence of keratin, which may be the reason for decreased antifungal activity of the compound inside nail plate (H. Tachibana et. al. (2017)). Thus, new antifungal compounds have been explored for with combination of properties including high penetration, low surface tension formulation, low affinity to keratin and different action mechanisms. The examples of such compounds have been summarized in Table 2 .

\begin{tabular}{|c|c|c|c|}
\hline $\begin{array}{l}\text { Antifungal } \\
\text { Compound } \\
\text { (Trade Name) }\end{array}$ & $\begin{array}{l}\text { Structural } \\
\text { Features }\end{array}$ & Action Mechanism & Properties \\
\hline Efinaconazole & Imidzole & $\begin{array}{l}\text { Inhibition of } \\
\text { ergosterol synthesis }\end{array}$ & $\begin{array}{c}\text { Low surface tension, } \\
\text { high penetration in } \\
\text { nail plate }\end{array}$ \\
\hline Tavaborole & $\begin{array}{c}\text { Boron based } \\
\text { oxaborole } \\
\text { molecule }\end{array}$ & $\begin{array}{l}\text { Selective inhibition of } \\
\text { fungal protein } \\
\text { synthesis process }\end{array}$ & $\begin{array}{l}\text { Affinity to fungal } \\
\text { enzymes, low } \\
\text { molecular weight and } \\
\text { easy nail penetration }\end{array}$ \\
\hline Auriclosene & Aganoside & $\begin{array}{l}\text { Nanoemulsion based } \\
\text { lacquers for growth } \\
\text { inhibition of } \\
\text { dermatophytes }\end{array}$ & Drug penetration \\
\hline ME1111 & $\begin{array}{l}\text { Pyrazol based } \\
\text { methyl phenol }\end{array}$ & $\begin{array}{c}\text { Targets fungal } \\
\text { electron transport } \\
\text { chain }\end{array}$ & $\begin{array}{l}\text { High nail penetration, } \\
\text { antifungal activity in } \\
\text { presence of keratin } \\
\text { not determined yet }\end{array}$ \\
\hline Luliconazole & Imidazole & $\begin{array}{l}\text { Inhibition of } \\
\text { ergosterol synthesis } \\
\text { and extracellular } \\
\text { protease secretion }\end{array}$ & $\begin{array}{c}\text { Low affinity with } \\
\text { keratin }\end{array}$ \\
\hline $\begin{array}{c}\text { Non oral } \\
\text { terbinafine } \\
\text { alternatives (MOB- } \\
\text { 015, P-3058 and } \\
\text { HTU-520) }\end{array}$ & Tertiary amine & Not known & $\begin{array}{l}\text { Improved transport } \\
\text { through nail barrier }\end{array}$ \\
\hline
\end{tabular}

The antifungal compounds provide systemic treatment measures for onychomycosis; however their efficiency to penetrate nail plate and efficacy in presence of keratin needs to be extensively studied. The reduced penetration results in alteration of MIC (minimum inhibitory concentration) reaching beneath the nail plate or in mass of nail debris. The morphological changes in diseased nail should not affect the efficiency of antifungal topical drug. Moreover, there is a need to conduct detailed studies on efficacy of these compounds according to stages of fungal agent such as spores which are mostly resistant to such treatments. The topical treatments are mostly used but long time of consistent practice leads to visible positive changes in the diseased nail. Thus, several other therapies have been devised to provide dermatological treatments at relatively less time period.

2.2. Laser treatments: The limitations of topical antifungal compounds in terms of nail plate penetration and inefficiency because of keratin developed lacuna for testing other therapies against onychomycosis. The oral antifungal compounds have also been reported to exhibit side effects on liver and kidney toxicity due to indirect drug interactions within the body (H.I.Katz et. al. (1999)). Since the discovery of laser technology, long pulse YAG (neodymium-doped yttrium aluminum garnet) lasers, short pulsed YAG lasers, CO2 lasers have emerged as new therapies for onychomycosis treatment. Drug therapies are usually consistently required for at least a year; laser therapy in onychomycosis is known to cut down the duration in half. The term laser refers to stimulated emission of radiation resulting into light amplification. The exact mechanism of laser radiations in onychomycosis remains unclear, but it can be expected that heat generation by lasers can disrupt fungal structures. Studies have shown that $1064 \mathrm{~nm}$ wavelength of light has the ability to efficiently penetrate the nail bed, where fungal material is exposed to heat inhibiting fungal growth, causing fungal cell damage, disrupting fungal 
mitochondrial membrane potential leading to inhibition of fungal growth rate (U. Kimura et. al. (2012).

Figure 3: Effect of laser therapy on onychomycosis. a) Pictorial representation of laser therapy over toe nail. b) Effect of laser treatment on fungi grown over agar plates where $L$ represents fungi growth before laser treatment and $R$ represents fungi growth after 3 days of laser treatment.c) Effect of short pulse Nd:YAG laser procedure over infected toe nail showing increased area of clearance. d) Percentage of nail clearance observed in comparison with infected area at the baseline(J. Kozarev (2010), D.P.M Chris Horine).

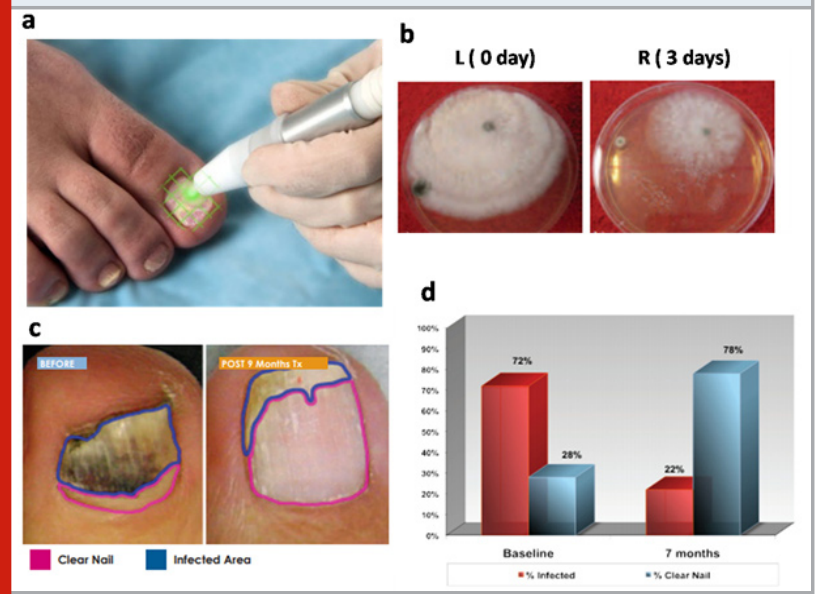

A study conducted by Horine et al., 262 patients received one PinPointe short pulsed Nd: YAG $1064 \mathrm{~nm}$ laser at 1.5 mm spot (pulse train ten $200 \mathrm{~mJ}, 90 \mu$ s duration, $1064 \mathrm{~nm}$ ). High speed blurr was used for pretreatment of debarment and thinning of hyperkeratotic nail plate. Comparative photographs demonstrated $100 \%$ response rate with $78 \%$ of the deformed nail clearup within seven months (S. Ladha et. al. (2019)). Another study conducted by Kozarev et al., long pulse Nd:YAG laser therapy was conducted in 72 patients diagnosed positive for onychomycosis andit was found that after 3 months follow up, 95.8\% patients reported clearance of infection. After 6 and 12 months follow up, $100 \%$ patients showed fungal infection clearance in form of nails free from hyperkeratosis and debris (J. Kozarev et. al. (2010)). Although the procedure is simple and quick without any probable negative effects, however, the mechanism of action still needs to be explored. Also, it is possible that patients may refrain from allowing laser radiations exposure in their body as inappropriate use of practice can lead to unnecessary radiation exposure (Figure 3 ).

\section{CONCLUSION}

Onychomycosis is a disease commonly observed as nail deformities spreading towards skin. The disease prevalence has spread worldwide owing to several problems in treatment strategies. The histological experiments reveal fungal spores present deep beneath the nail bed making the disease more complex. The most commonly used treatments include topical antifungal agents but limitations exist in terms of inability to penetrate the dead cell mass of hyperkeratotic nail. The treatment procedures developed to treat onychomycosis include laser treatments which cause observational changes in the nail in less time. The disease treatment often cause relapse due to penetration of pathogen in surrounding tissue.

Moreover, patients refrain from laser treatments as it causes radiation exposure if not done with expertise. Thus there is a need to develop novel strategies to for treating onychomycosis. Device based inventions such as sealed patch containing antifungal compounds can reduce progression of the infection towards surrounding tissue. Alternatively, nanoparticles loaded with antifungal compounds can be developed to induce targeted drug delivery inside the nail bed. Overall, it is required to develop innovative strategies to minimize disease relapse and progression to areas beyond nails. This study elaborately discusses onychomycosis from pathological and clinical perspective and provides open research horizons for device based discoveries in treatment of onychomycosis.

\section{REFERENCES}

Adekhandi, S., Pal, S., Sharma, N., Juyal, D., Sharma, M. and Dimri, D., 2015. Incidence and epidemiology of onychomycosis in patients visiting a tertiary care hospital in India. Cutis, 95(1), pp.E20-5.

Chris Horine, D.P.M., Dow, H., DPodM, H.C.P.C. and John Cozzarelli, D.P.M., Evaluation of a short pulse Nd: YAG Laser Procedure for Infected Nails - A Retrospective International Multicenter Study of 262 Patients.

Elewski, B.E. and Tosti, A., 2015. Risk factors and comorbidities for onychomycosis: implications for treatment with topical therapy. The Journal of clinical and aesthetic dermatology, 8(11), p.38.

Ezhilarasi, G.D., Latchoumi, T.P. and Balamurugan, K., 2020. UIP_A Smart Web Application to Manage Network Environments, Advances in Intelligent systems and computing book series.

Ghannoum, M. and Isham, N., 2014. Fungal nail infections (onychomycosis): a never-ending story? PLoS pathogens, 10(6), p.e1004105.

Gupta, A.K. and Elewski, B.E., 1996. Nondermatophyte causes of onychomycosis and superficial mycoses. Current topics in medical mycology, 7(1), pp.87-97.

Katsambas, A.D., Lotti, T.M., Dessinioti, C. and D'Erme, A.M. eds., 2015. European handbook of dermatological treatments. Springer.

Katz, H.I., 1999. Drug interactions of the newer oral antifungal agents. The British journal of dermatology, 141, pp.26-32.

Kawa, N., Lee, K.C., Anderson, R.R. and Garibyan, L., 2019. Onychomycosis: A review of new and emerging topical and device-based treatments. The Journal of clinical and aesthetic dermatology, 12(10), p.29.

Kozarev, J. and Vizintin, Z., 2010. Novel laser therapy in 
treatment of onychomycosis. J Laser Health Acad, 1(1), pp.1-8.

Mishra, P., Jimmy, L., Ogunmola, G.A., Phu, T.V., Jayanthiladevi, A. and Latchoumi, T.P., 2020, December. Hydroponics cultivation using real time iot measurement system. In Journal of Physics: Conference Series (Vol. 1712, No. 1, p. 012040). IOP Publishing.

Nada, E.E.D.A., El Taieb, M.A., El-Feky, M.A., Ibrahim, H.M., Hegazy, E.M., Mohamed, A.E. and El-Amir, M.I., 2020. Diagnosis of onychomycosis clinically by nail dermoscopy versus microbiological diagnosis. Archives of dermatological research, 312(3), pp.207-212.

Namekar, M., Ellis, E.M., O'Connell, M., Elm, J., Gurary, A., Park, S.Y., Imrie, A. and Nerurkar, V.R., 2013. Evaluation of a new commercially available immunoglobulin M capture enzyme-linked immunosorbent assay for diagnosis of dengue virus infection. Journal of clinical microbiology, 51(9), pp.3102-3106.

Prasath, S. (2020). Probabilistic Mceliece Public-Key Cryptography Based Identity Authentication For Secured Communication In Vanet. Solid State Technology, 63(6), 10167-10182.

Prasath, S. Validating Data Integrity in Steganographed Images using Embedded Checksum Technique.
International Journal of Computer Applications, 975, 8887.

Tachibana, H., Kumagai, N. and Tatsumi, Y., 2017. Fungicidal activity in the presence of keratin as an important factor contributing to in vivo efficacy: a comparison of efinaconazole, tavaborole, and ciclopirox. Journal of Fungi, 3(4), p.58.

Queller, J.N. and Bhatia, N., 2015. The dermatologist's approach to onychomycosis. Journal of Fungi, 1(2), pp.173-184.

Utako Kimura, M., Kaori Takeuchi, M., Ayako Kinoshita, M., Kenji Takamori, M. and Masataro Hiruma, M.D., 2012. Treating onychomycoses of the toenail: clinical efficacy of the sub-millisecond $1064 \mathrm{~nm}$ nd: yag laser using a $5 \mathrm{~mm}$ spot diameter. J Drugs Dermatol, 11(4), pp.496-504.

Venkata Pavan, M., Karnan, B. and Latchoumi, T.P., 2021. PLA-Cu reinforced composite filament: Preparation and flexural property printed at different machining conditions. Advanced Composite Materials, https://doi. org/10.1080/09243046.2021, 1918608.

Westerberg, D.P. and Voyack, M.J., 2013. Onychomycosis: current trends in diagnosis and treatment. American family physician, 88(11), pp.762-770. 